Dušan I. Ilić ${ }^{\text {, Jovan P. Šetrajčić }}{ }^{2 *}$

${ }^{1}$ University of Novi Sad, Faculty of Technical Sciences, Chair of Physics, Novi Sad, Serbia

${ }^{2}$ University of Novi Sad, Faculty of Sciences, Department of Physics, Novi Sad, Serbia
Scientific paper

ISSN 0351-9465, E-ISSN 2466-2585

UDC:620.197.16

doi:10.5937/ZasMat1803442I

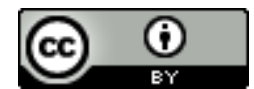

Zastita Materijala 59 (3)

442 - 453 (2018)

\title{
Influence of the phonon subsystem onto the mechanical and thermodynamic properties of low-dimensional materials
}

\begin{abstract}
This paper provides an overview of the influence of the phonon subsystem onto the mechanical and thermodynamic properties of low-dimensional materials (particularly, ultrathin films), which has been the subject of our team's research for many years. Explorations of that kind have become very important for the materials research science and engineering over the last few years, due to the great commercialization potential of the novel two-dimensional structures, such as graphene. It is shown how the quantum size effect influences the change of physical properties of the above-mentioned structures and the consequences of this impact have been discussed in detail. Theoretical analysis has been conducted by means of the method of two-time dependent Green's functions by which the spectra of allowed phonon energies, as well as thermodynamic characteristics of these structures, has been determined. The diffusion processes have been treated by means of Kubo formula, adapted to the phonon subsystems in nanostructures.
\end{abstract}

Keywords: Phonons, Green's function, ultrathin films, specific heat, diffusion tensor.

\section{INTRODUCTION}

Researching mechanical and thermodynamic properties of nanostructures represents one of the greatest scientifical challenges in recent years. Thanks to the exceptional possibilities of applying low-dimensional systems in various fields of science, technology, and human life in general, the examination of these materials and structures have become one of the cornerstones of modern science. This particularly applies to the novel twodimensional crystalline structures such as graphene, being the most exciting and amazing material for potential application of today. But it is precisely on the example of graphene that has been shown to which extent ignorance of the basic physical properties of the nanostructures limits the possibilities of their utilization. It is now rightly understood that only with the thorough and serious theoretical and experimental examination of nanostructures, the full appreciation of their possibilities can be achieved. The central aim of this paper is to show how dimensional limitation of

\footnotetext{
${ }^{*}$ Corresponding author: Jovan P. Šetrajčić

E-mail: jovan.setrajcic@df.uns.ac.rs

Paper received: 31. 03. 2018.

Paper accepted: 29. 04. 2018.

Paper is available on the website: www.idk.org.rs/journal
}

phonons in low-dimensional structures (ultrathin films, in particular, but the same theoretical approach can without any reservations be applied to the other size-limited structures) influences its mechanical and thermodynamic properties, which creates the foundation for the idea of phonon engineering (nano-phononics) [1-5]. In accordance with a theoretically almost limitless range of applications of ultrathin films, graphene, in particular, it is necessary to fully understand the physical principles that make them so special.

Why fonons? It is well-known fact that the phonon subsystem represents one of the most important always present systems in condensed matter that affects acoustic, thermal, as well as conductive and superconductive properties of materials. Phonon interactions with electrons, excitons, and other elementary excitations to a large extent determine and affect the state of the system under consideration. It has been shown that the spatial confinement of phonons, as a result of the quantum size effect, greatly changes the physical properties of the materials. The concept of phonon engineering is based exactly on these changes in order to improve mechanical, electric and thermodynamic properties of materials by changing the size of the sample, by inserting atoms of different kinds or by changing the quality of exterior surfaces. Given the enormous importance 
that the heat removal has for the operation and reliability of electronic devices, it becomes clear how valuable is the ability to create the desired physical properties by changing some of these parameters.

\section{PHONONS IN A MACROSCOPIC SIZE CRYSTALS}

\subsection{Phonon dispersion law}

By expanding the potential energy of crystals in a sum by small atomic displacements from the equilibrium position $\vec{u}_{\vec{n}}$, one obtains the Hamiltonian of the phonon subsystem in a crystalline structure under consideration. Here, $\vec{n}=n_{x} \vec{a}_{x}+n_{y} \vec{a}_{y}+n_{z} \vec{a}_{z}$ is the vector of the crystal lattice, where $\vec{a}_{x}, \vec{a}_{y}$ and $\vec{a}_{z}$ are vectors of the crystal unit cell, and $n_{x}, n_{y}, n_{z}$ are integers. Only crystals with a simple cubic crystalline structure containing one atom per elementary cell will be considered in this review. Although at first, this seems like a vast restriction in terms of applicability of chosen model structure, it turns out this is not the case: in fact, in accordance with the method of achievement statistical and dynamical equivalence between rectangular and structures with lower symmetry [4], this model has potential to be suitable in many cases. Hamiltonian of the phonon subsystem in such structures, displayed in the nearest neighbor approximation can be written in the following form [6-7]:

$$
\begin{aligned}
& H=\sum_{\alpha ; \vec{n}} \frac{p_{\alpha ; \vec{n}}^{2}}{2 M}+\sum_{\alpha ; n_{x}, n_{y}, n_{z}} \frac{C^{\alpha}}{4}\left[\left(u_{\alpha ; n_{x}+1, n_{y}, n_{z}}-u_{\alpha ; n_{x}, n_{y}, n_{z}}\right)^{2}+\left(u_{\alpha ; n_{x}-1, n_{y}, n_{z}}-u_{\alpha ; n_{x}, n_{y}, n_{z}}\right)^{2}+\right. \\
& +\left(u_{\alpha ; n_{x}, n_{y}+1, n_{z}}-u_{\alpha ; n_{x}, n_{y}, n_{z}}\right)^{2}+\left(u_{\alpha ; n_{x}, n_{y}-1, n_{z}}-u_{\alpha ; n_{x}, n_{y}, n_{z}}\right)^{2}+ \\
& \left.+\left(u_{\alpha ; n_{x}, n_{y}, n_{z}+1}-u_{\alpha ; n_{x}, n_{y}, n_{z}}\right)^{2}+\left(u_{\alpha ; n_{x}, n_{y}, n_{z}-1}-u_{\alpha ; n_{x}, n_{y}, n_{z}}\right)^{2}\right] \text {, }
\end{aligned}
$$

where $M$ is the mass of the atoms that constitute the crystalline lattice, $u_{\alpha ; \vec{n}}$ are the small displacements of an atom in the node $\vec{n}$ from its equilibrium position in direction $\alpha$, and $\vec{p}_{\alpha ; \vec{n}}$ the phonon momentum. Hooke's torsion constants are neglected in comparison to the tension constant.

In order to derive the phonon dispersion law in crystalline structures, we start from phonon twotime commutator Green's function [8]:

$$
G_{\vec{n}, \vec{m}}^{\alpha}\left(t-t^{\prime}\right) \equiv\left\langle\left\langle u_{\alpha ; \vec{n}}(t) \mid u_{\alpha ; \vec{m}}\left(t^{\prime}\right)\right\rangle\right\rangle=\Theta\left(t-t^{\prime}\right)\left\langle\left[u_{\alpha ; \vec{n}}(t), u_{\alpha ; \vec{m}}\left(t^{\prime}\right)\right]\right\rangle_{0}
$$

for which we formulate the equation of motion:

$$
M \frac{d^{2}}{d t^{2}} G_{\vec{n}, \vec{m}}^{\alpha}\left(t-t^{\prime}\right)=-i \hbar \delta_{\vec{n}, \vec{m}} \delta\left(t-t^{\prime}\right)+\frac{\Theta\left(t-t^{\prime}\right)}{i \hbar}\left\langle\left[\left[p_{\alpha ; \vec{n}}(t), H(t)\right], u_{\alpha ; \vec{m}}\left(t^{\prime}\right)\right]\right\rangle_{0} .
$$

Performing Fourier transform $t \rightarrow \omega$, the last equation converts into:

$$
-M \omega^{2} G_{\vec{n}, \vec{m}}^{\alpha}(\omega)=-\frac{i \hbar}{2 \pi} \delta_{\vec{n}, \vec{m}}+\frac{1}{i \hbar}\left\langle\left[\left[p_{\alpha ; \vec{n}}, H\right], u_{\alpha ; \vec{m}}\right]\right\rangle_{\omega} .
$$

In the next step to determining Green's functions, we have to calculate a commutator that appears in higher-order Green's functions in Eq. (4). By performing spatial Fourier transform $\vec{n}, \vec{m} \rightarrow \vec{k}:$

$$
\begin{aligned}
& G_{\vec{n}, \vec{m}}^{\alpha}(\omega)=\frac{1}{N} \sum_{\vec{k}} e^{-i(\vec{n}-\vec{m}) \vec{k}} G_{\vec{k}}^{\alpha}(\omega) ; \\
& \delta_{\vec{n}, \vec{m}}=\frac{1}{N} \sum_{\vec{k}} e^{-i(\vec{n}-\vec{m}) \vec{k}}
\end{aligned}
$$

and after few simple algebraic steps, the last equation converts into:

$$
G_{\vec{k}}^{\alpha}(\omega)=\frac{i \hbar}{4 \pi M \omega_{\alpha}(\vec{k})}\left[\frac{1}{\omega-\omega_{\alpha}(\vec{k})}-\frac{1}{\omega+\omega_{\alpha}(\vec{k})}\right]
$$

where $\omega_{\alpha}(\vec{k})=2 \Omega_{\alpha} \sqrt{\sum_{j} \sin ^{2} \frac{a_{j} k_{j}}{2}}, j \in(x, y, z)$ and $\Omega_{\alpha}=\sqrt{C_{\alpha} / M}$. From this equality, it can be seen that the poles of Green's functions are determined when the denominator of expression in brackets equalizes with zero. By solving this condition, taking $\omega \equiv \omega_{\alpha}(\vec{k})$, the phonon dispersion law in macroscopic size crystals is obtained in the form: 


$$
\begin{aligned}
& E_{\alpha}(\vec{k}) \equiv \hbar \omega_{\alpha}(\vec{k})= \\
& =2 E_{\alpha} \sqrt{\sin ^{2} \frac{a_{x} k_{x}}{2}+\sin ^{2} \frac{a_{y} k_{y}}{2}+\sin ^{2} \frac{a_{z} k_{z}}{2}}
\end{aligned}
$$

where $E_{\alpha}=\hbar \Omega_{\alpha}=\hbar \sqrt{C_{\alpha} / M}$. In order to relate this equation with that for ultrathin film structures, it is useful to rewrite it in the form:

$$
\mathscr{C}_{\alpha}(\vec{k}) \equiv \frac{E_{\alpha}(\vec{k})}{E_{\alpha}}=2 \sqrt{\mathscr{T}\left(k_{x} k_{y}\right)+\mathscr{g}\left(k_{z}\right)}
$$

or, in a simpler manner:

$$
\mathscr{C}_{x y z}=2 \sqrt{\mathscr{F}_{x y}+\mathscr{C}_{z}}
$$

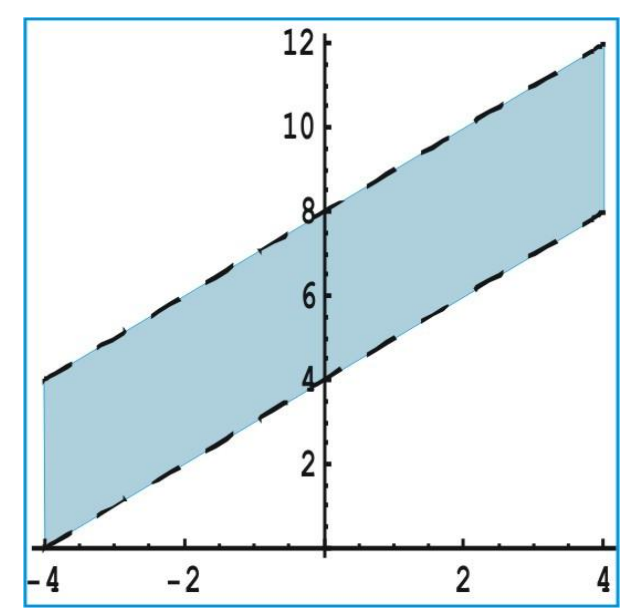

Figure 1. Phonon dispersion law for bulk structure

Slika 1. Zakon disperzije fonona u balku

All these energies are equally probable and their spatial distribution in the crystal is even. This is an understandable consequence of the absence of boundary conditions and imperfections of the structure.

\subsection{Phonon-induced thermodynamic properties}

We will assume that phonon wave vectors are arranged within the sphere of radius $k_{D}$ rather than in the first Brillouin zone $\left(k_{D}\right.$ is the Debye wave vector). Starting from this assumption, it can be written:

The internal energy of the system is calculated by standard definitional form [6-7]:

$$
U=\int_{0}^{\omega_{D}} d \omega\left(\frac{\omega^{2} V}{2 \pi^{2} v^{3}}\right)\left(\frac{\hbar \omega}{e^{\frac{h \omega}{k_{B} T}}-1}\right)=\frac{V}{2 \pi^{2} v^{3} \hbar^{3}}\left(k_{B} T\right)^{4} \int_{0}^{\omega_{D}} \frac{\left(\frac{h \omega}{k_{B} T}\right)^{3}}{e^{\frac{h \omega}{k_{B} T}}-1} d\left(\frac{h \omega}{k_{B} T}\right)
$$

If, for the sake of simplicity, we assume that the phonon velocity does not depend on polarization, it follows: where:

$$
\begin{aligned}
& \mathscr{F}_{x y} \equiv \mathscr{F}\left(k_{x} k_{y}\right)=\sin ^{2} \frac{a_{x} k_{x}}{2}+\sin ^{2} \frac{a_{y} k_{y}}{2} ; \\
& \mathscr{C}_{z} \equiv \mathscr{C}\left(k_{z}\right)=\sin ^{2} \frac{a_{z} k_{z}}{2}
\end{aligned}
$$

Figure 1 shows the dependence of the square on the relative phonon energy $c_{x y z}^{2}$ in terms of the two-dimensional function $\mathscr{F}_{x y}$ with a parametric function $/ / 2$ for the first Brillouin zone of the bulk structures. The dotted lines indicate the boundaries of the bulk zone. It can be seen that within the permissible energy zone, there is the same number of possible energy states, as the number of atoms within the crystal lattice.

$$
\begin{gathered}
V=\left\{\begin{array}{c}
\frac{4}{3} \pi\left(a k_{D}\right)^{3} \\
a^{3} \Delta k_{x} \Delta k_{y} \Delta k_{z} ;
\end{array}\right. \\
k_{x / y / z} \in\left[-\frac{\pi}{a},+\frac{\pi}{a}\right] \Rightarrow \Delta k_{x}=\Delta k_{y}=\Delta k_{z}=\frac{2 \pi}{a}
\end{gathered}
$$

on the basis of which it follows:

$$
\frac{4}{3} \pi\left(a k_{D}\right)^{3}=a^{3} \Delta k_{x} \Delta k_{y} \Delta k_{z}=8 \pi^{3} \Rightarrow k_{D}=\frac{\sqrt[3]{6 \pi^{2}}}{a}
$$

and the Debye frequency is:

$$
\omega_{D}=k_{D} \cdot v=\frac{\sqrt[3]{6 \pi^{2}}}{a} \cdot v
$$

where $v$ is the speed of sound in the crystal. Through the Debye frequency, the corresponding Debye temperature $T_{D}$ is also introducing:

$$
k_{B} T_{D}=\hbar \omega_{D} \Rightarrow T_{D}=\frac{\hbar \omega_{D}}{k_{B}}=\frac{\hbar k_{D} v}{k_{B}}=\frac{\sqrt[3]{6 \pi^{2}} \hbar v}{k_{B} a}
$$

where $k_{B}$ is the Boltzmann constant. 


$$
U=9 N k_{B} T\left(\frac{T}{T_{D}}\right)^{3} \int_{0}^{x_{D}} \frac{x^{3}}{e^{x}-1} d x
$$

where $N$ is the number of atoms in the sample, $x=\frac{\hbar \omega}{k_{B} T}$ and $x_{D}=\frac{\hbar \omega_{D}}{k_{B} T}$. Thermal capacitance is obtained by differentiating the previous equation by temperature:

$$
C=9 N k_{B} T\left(\frac{T}{T_{D}}\right)^{3} \int_{0}^{x_{D}} \frac{x^{4} e^{x}}{\left(e^{x}-1\right)^{2}} d x
$$

In the case of high temperatures, when $T \gg T_{D}$, the previous equation is reduced to the Dulong-Petit law. In the low-temperature region, when $x_{D} \rightarrow \infty$, equation (12) becomes:

$$
U=\frac{3 \pi^{4}}{5} N k_{B} T\left(\frac{T}{T_{D}}\right)^{3}
$$

so that the expression for thermal capacitance in this case takes the form:

$$
C=\frac{12 \pi^{4}}{5} N k_{B}\left(\frac{T}{T_{D}}\right)^{3}
$$

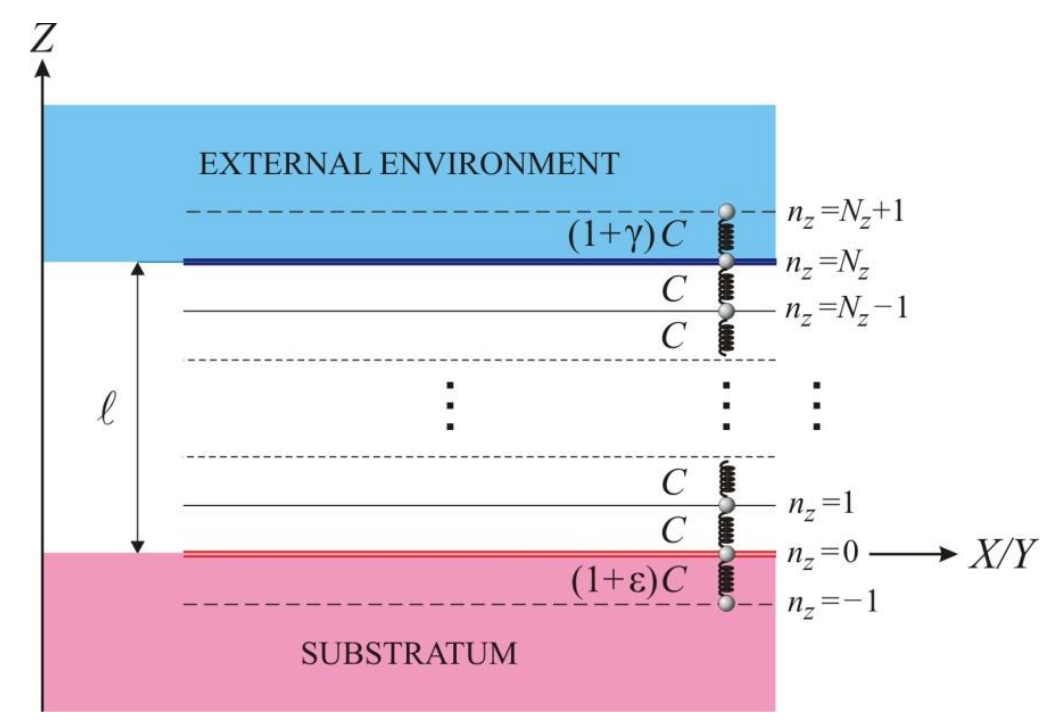

Figure 2. The crystalline ultrathin film model-structure

Slika 2. Model razmatranog ultratankog kristalnog filma

The main consequence of symmetry disturbance is the spatial dependence of physical properties of the system. In the considered case, physical properties will depend on the index $n_{z}=0,1,2, \ldots, N_{z} ; 2 \leq N_{z} \leq 10$, labelling layers of the film in $z$-direction. Spatial dependence is determined by boundary conditions. Here the which is known as Debye $T^{3}$ - law. In order to establish a connection between the thermal capacitance of massive crystal structures with those of ultrathin films, we will divide the previous equation with $N$ to obtain the thermal capacitance calculated by one elementary cell of the crystal:

$C^{*}=\frac{1}{N} \frac{\partial U}{\partial T}=\frac{12 \pi^{4}}{5} k_{B}\left(\frac{T}{T_{D}}\right)^{3} \equiv C_{0} \cdot \mathscr{T}^{3}$

where $C_{0} \equiv \frac{12 \pi^{4}}{5} k_{B}$ and $\mathscr{T} \equiv \frac{T}{T_{D}}$.

\section{A BUILDING OF THE PHONON MODEL IN ULTRATHIN FILMS}

Ultrathin films [5,9-11] are confined crystalline structures in which the boundary conditions differ from those in the interior, i.e. in which translational symmetry is disturbed along the direction perpendicular to the film. The model we have chosen for this analysis is the model of ideal (on boundary planes - nonperturbed) ultrathin film which is infinite in $X Y$ planes and has a finite (nano-small) thickness in the $z$-direction. It means that symmetry disturbance appears on surfaces in $z$-direction (Fig.2). simplest boundary conditions will be taken consisting in the absence of layers labelled with $n_{z}=-1$ and $n_{z}=N_{z}+1$.

The analysis of the film-properties will be carried out with the help of Green's functions of displacement as well as momentum types and their spectral intensities will be used for determination of 
some relevant kinetic and thermodynamic characteristics of the film. The method of Green's functions is generally used here because it is selfconsistent, i.e. it solves both the dynamic and thermodynamic aspects of the problem. Different methods are used for solving one or the other problem. For example, dynamic characteristics can be determined by using the wave function, but in order to solve thermodynamics, one has to resort to methods of statistical physics.

The basic crystallographic parameters of the chosen model structure - in the nearest neighbor approximation - are:

$$
\begin{aligned}
& a_{x}=a_{y}=a_{z} \equiv a ; \quad N_{x, y} \sim 10^{8}>N_{z} \sim 10, \\
& C_{\vec{n}, \vec{m}}^{\alpha, \beta}=C_{\vec{n}, \vec{m}}^{\alpha, \alpha}=C_{\vec{n}, \vec{n} \pm \vec{\lambda}}^{\alpha}=C_{\vec{n}, \vec{n} \pm \vec{\lambda}}=C_{n_{z}, n_{z} \pm 1}, \\
& C_{N_{z}+1, N_{z}}=C_{N_{z}, N_{z}+1}=(1+\gamma) C, \\
& C_{-1,0}=C_{0,-1}=(1+\varepsilon) C ; \\
& \varepsilon, \gamma \in[-1,+1,5] .
\end{aligned}
$$

where $a$ is the constant of the crystal lattice, $N_{x, y, z}$ are the numbers of atoms along $x, y$ and $z$ directions, $C^{\alpha}$ is the straining Hooke's elastic constant in direction $\alpha, \quad$ and $n_{x, y, z} \in\left(0,1,2, \ldots, N_{x, y, z}\right)$.

Having in mind all that has been said above, we can bring the following conclusions about the selected model structure:

- the crystalline film has two infinite boundaries parallel to the $X Y$ planes for $z=0$ and $z=L$, while along $z$-direction it possesses finite thickness $\left(L=N_{z} a\right)$. Along $z$-direction there are $N_{z}+1$ atoms;
- torsion Hooke's elastic constants $C^{\alpha \beta}$ are negligible relative to the straining constants $C^{\alpha \alpha} \equiv C^{\alpha}$ :

$C_{\vec{n}, \vec{m}}^{\alpha,}=C_{\vec{n}, \vec{n} \pm \vec{\lambda}}^{\alpha}=C_{\vec{n}, \vec{n} \pm \vec{\lambda}}=C_{n_{z}, n_{z^{ \pm}} 1}=C_{n_{z^{ \pm}}}$.

It is considered that there is an interaction between atoms in boundary layers of the film and external areas (substrate and e.g. air), disregarding that along $z$-direction there are no atoms belonging to the film; however, boundary atoms are coupled through changed Hooke's forces with the atoms of the external environment [12-19]. In accordance with these conditions, elastic constants which describe the interaction between atoms of boundary surfaces and external environment are modified with appropriate coefficients $\varepsilon$ and $\gamma$. With respect to described model and concerning the fact that layers with $n_{z} \leq-1$ and $n_{z} \geq N_{z}+1$ are missing, we have to reckon the following: $u_{\alpha ; n_{x}, n_{y}, j}=0, \quad-1 \geq j \wedge j \geq N_{z}+1, \quad j \notin\left[0, N_{z}\right]$, $C_{-1}=(1+\varepsilon) C ; \quad C_{N_{z}+1}=(1+\gamma) C$. If it were: $C_{-1}=C_{N_{z}+1}=0 \quad(\varepsilon=\gamma=-1)$, then the boundary atoms for $n_{z}=0$ and $n_{z}=N_{z}$ would be "frozen", that is, the effect of "rigid walls" would have appeared. On the other hand, in the case where it is $C_{-1}=C_{N_{z^{+1}}}=C \quad(\varepsilon=\gamma=0)$, it is the effect of "free surfaces". In this paper we have opted for the latter case of flexible boundary surfaces, considering that this is closer to the real situation in which the ultrathin film can "breathe".

Hamiltonian of the phonon subsystem for described ultrathin crystalline film - written in the nearest neighbor approximations and adapted to the ultrathin film model structure presented in Fig. 2 - is:

$$
\begin{aligned}
H=\sum_{\alpha ; n} & \frac{p_{\alpha ; n}^{2}}{2 M}+\sum_{\alpha, n_{x}, n_{y}} \sum_{n_{z}=-1}^{N_{z}^{+1}} \frac{C_{n_{z}}^{\alpha}}{4}\left[\left(u_{\alpha ; n_{x}+1, n_{y}, n_{z}}-u_{\alpha ; n_{x}, n_{y}, n_{z}}\right)^{2}+\left(u_{\alpha ; n_{x}-1, n_{y}, n_{z}}-u_{\alpha ; n_{x}, n_{y}, n_{z}}\right)^{2}+\right. \\
& +\left(u_{\alpha ; n_{x}, n_{y}+1, n_{z}}-u_{\alpha ; n_{x}, n_{y}, n_{z}}\right)^{2}+\left(u_{\alpha ; n_{x}, n_{y}-1, n_{z}}-u_{\left.\alpha ; n_{x}, n_{y}, n_{z}\right)^{2}+}\right. \\
& \left.+\left(u_{\alpha ; n_{x}, n_{y}, n_{z}+1}-u_{\alpha, n_{x}, n_{y}, n_{z}}\right)^{2}+\left(u_{\alpha ; n_{x}, n_{y}, n_{z}-1}-u_{\alpha ; n_{x}, n_{y}, n_{z}}\right)^{2}\right] .
\end{aligned}
$$

Allowed phonon energies will be determined by following the same procedure described for the massive crystalline samples and by applying the partial spatial Fourier-transformation along $z-$ direction, where the translational symmetry has been interrupted. In this way, we get the system of $\left(N_{z}+1\right)$ nonhomogeneous algebraic-difference equations with the same number of undetermined Green's functions [5-19]:

$$
G_{n_{z}-1, m_{z}}^{\alpha}+\rho_{k}^{\alpha} G_{n_{z}, m_{z}}^{\alpha}+G_{n_{z}+1, m_{z}}^{\alpha}=K \delta_{n_{z}, m_{z}}
$$

where:

$$
G_{n_{2}, m_{z}}^{\alpha} \equiv G_{n_{2}, m_{z}}^{\alpha}\left(k_{x} k_{y} ; \omega\right) ; K \equiv \frac{i \hbar}{2 \pi C_{\alpha}} ; k \equiv \sqrt{k_{x}^{2}+k_{y}^{2}}
$$

while the determinant of that system of equations is: 


$$
D_{N_{z}+1}(\rho)=\left|\begin{array}{ccccccc}
\rho-\varepsilon & 1 & 0 & \ldots & 0 & 0 & 0 \\
1 & \rho & 1 & \ldots & 0 & 0 & 0 \\
0 & 1 & \rho & \ldots & 0 & 0 & 0 \\
. & . & . & \ddots & . & . & . \\
0 & 0 & 0 & \ldots & \rho & 1 & 0 \\
0 & 0 & 0 & \ldots & 1 & \rho & 1 \\
0 & 0 & 0 & \ldots & 0 & 1 & \rho-\gamma
\end{array}\right|_{N_{z}+1}
$$

where:

$$
\rho_{k}^{\alpha}=\frac{\omega^{2}}{\Omega_{\alpha}^{2}}-4 \sin ^{2} \frac{a k_{x}}{2}-4 \sin ^{2} \frac{a k_{y}}{2}-2 \equiv \rho
$$

Spectra of the possible phonon energies is determined by finding of the zeroes of the determinant (17), i.e. by solving the equality:

$$
\begin{aligned}
& D_{N_{z}+1}(\rho ; \varepsilon, \gamma) \equiv 0 \Rightarrow \rho=\rho_{v}(\varepsilon, \gamma) ; \\
& v=1,2,3, \ldots N_{z}+1 .
\end{aligned}
$$

This procedure gives the phonon dispersion law in an ultrathin, unperturbed crystalline film in the form:

$$
\begin{aligned}
& \quad C_{v}^{\alpha}(\vec{k}) \equiv \frac{E_{\alpha}(\vec{k})}{E_{\alpha}}=2 \sqrt{\sin ^{2} \frac{a_{x} k_{x}}{2}+\sin ^{2} \frac{a_{y} k_{y}}{2}+\sin ^{2} \frac{a_{z} k_{z}(v)}{2}} \\
& \text { or: } \mathscr{C}_{v}=2 \sqrt{\widetilde{\sigma}_{x y}+\mathscr{C}_{v}}
\end{aligned}
$$

where $v=1,2, \ldots, N_{z}+1$. This equation is almost exactly the same as Eq. (6), with one significant difference: quasimomentum of phonons in ultrathin films can take only discrete values in $z$-direction,

while it is continual in $x$ - and $y$-directions within the interval $[0, \pi / a]$. Possible values for $k_{z}(v)$ are obtained by solving the transcendental equation:

$$
\operatorname{ctg}\left(N_{z}-1\right) a k_{z}(v)=\frac{4 \cos ^{3} a k_{z}(v)+2(\varepsilon+\gamma) \cos ^{2} a k_{z}(v)+(\varepsilon \gamma-3) \cos a k_{z}(v)+\varepsilon \gamma}{{\sin a k_{z}}_{z}(v)\left[2(\varepsilon+\gamma) \cos a k_{z}(v)-4 \cos ^{2} a k_{z}(v)-(1+\varepsilon \gamma)\right]}
$$

which in the "free surfaces" case diminishes to:

$$
k_{z}(v)=\frac{\pi}{a} \frac{v}{N_{z}+2}
$$

$$
\Delta \equiv \Delta_{\min }=\delta_{1}^{\alpha}\left(k_{x}=k_{y}=0, k_{z}=k_{z}^{\min }\right)=2 \sin \left[\frac{\pi}{2\left(N_{z}+2\right)}\right]
$$

By analyzing the phonon dispersion law for ultrathin films (20), it can be seen that:

$$
\begin{aligned}
& k_{x}^{\min }=k_{y}^{\min }=0, \\
& k_{z}^{\min } \equiv k_{z}(v=1)=\frac{\pi}{a} \frac{1}{N_{z}+2}>0,
\end{aligned}
$$

and:

$$
\begin{aligned}
& k_{z}^{\max }=k_{y}^{\max }=\frac{\pi}{a}, \\
& k_{z}^{\max } \equiv k_{z}\left(v=N_{z}+1\right)=\frac{\pi}{a} \frac{N_{z}+1}{N_{z}+2}<\frac{\pi}{a} .
\end{aligned}
$$

There are also $N_{z}-1$ discrete values between the minimum and maximum values for $k_{z}$, which means that the phonons in the ultrathin film possess a lower energy gap:

as well as - physically much less interesting upper energy gap. This minimum energy corresponds to the minimum frequency of phonons in an ultrathin crystalline film:

$\omega_{\min }\left(k_{x}=k_{y}=0, k_{z}=k_{z}^{\min }\right)=2 \frac{v}{a} \cdot \sin \left[\frac{\pi}{2\left(N_{z}+2\right)}\right] \neq 0$

The central outcome of this fact is that the zone of allowed phonon energies in ultrathin films is narrower than that in bulk samples for the value of the sum of these gaps. Gaps are clearly seen in Figure 3, where the phonon dispersion law (20) is shown: interrupted lines denote unbounded structures - where the spectra is continual, and solid lines indicate ultrathin film with discrete spectra. 


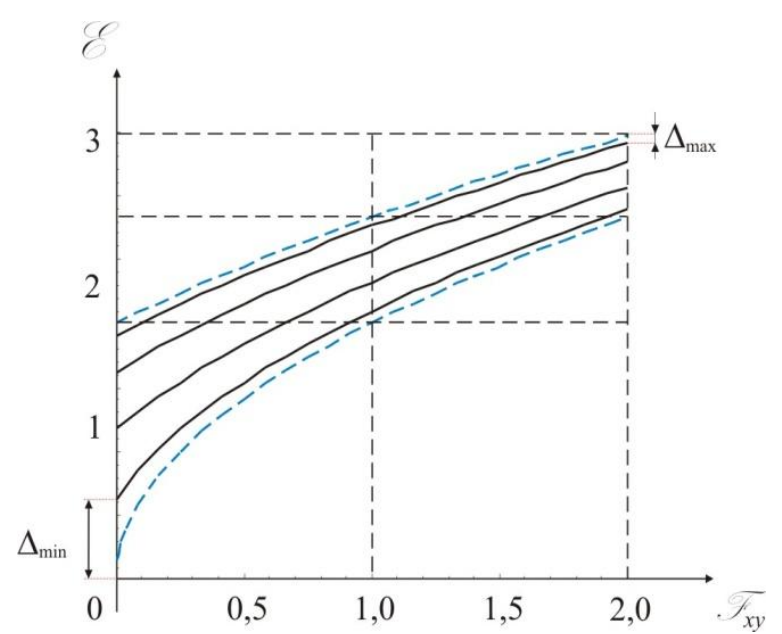

Figure 3. The phonon dispersion law for ultrathin film structure

Slika 3. Zakon disperzije fonona u ultratankom filmu

For the sake of a clearer comparison between the phonon dispersion laws in ultrathin films and unbounded crystalline structures, we will make use of the well known trigonometric relation:

$$
\sin ^{2} \alpha=\frac{1}{2}(1-\cos 2 \alpha)
$$

in order to rewrite Eq. (18) in the form:

$$
\rho=\frac{\omega^{2}}{\Omega_{\alpha}^{2}}-6+2\left(\cos a k_{x}+\cos a k_{y}\right)
$$

If we introduce tags:

$$
\begin{aligned}
& \frac{\omega^{2}}{\Omega_{\alpha}^{2}}-6 \equiv \sigma_{v}^{2} \text { and } \\
& 2\left(\cos a k_{x}+\cos a k_{y}\right) \equiv \mathscr{F}_{x y}
\end{aligned}
$$

Eq. (23) is reduced to: $\rho=\tau_{v}^{2}+\mathscr{F}_{x y}$

Figure 4 shows dependence $\tau_{v}^{2}=\tau_{v}^{2}\left(\mathscr{F}_{x y}\right)$ with a parametric function $\mathscr{C}_{v}$ for the five-layer perturbed film. Gaps and energy discreteness, as and therefore:

$$
V=\left\{\begin{array}{c}
\frac{4}{3} \pi\left(a k_{D}^{f}\right)^{3} \\
a^{3} \Delta k_{x} \Delta k_{y} \Delta k_{z}
\end{array} \Rightarrow k_{D}^{f}=\frac{\sqrt[3]{3 \pi^{2}}}{a} \cdot \sqrt[3]{\frac{N_{z}}{N_{z}+2}}=k_{D} \cdot \sqrt[3]{\frac{N_{z}}{2\left(N_{z}+2\right)}},\right.
$$

where $k_{D}$ is the phonon wave vector for bulk structures, Eq. (9). A density of phonon states in the ultrathin film is calculating by using the expression:

$$
D_{f}(\omega)=\frac{3 N_{x} N_{y} N_{z}^{f} a^{3}}{(2 \pi)^{3}} \int_{0}^{\pi} \sin \vartheta d \vartheta \int_{0}^{2 \pi} d \phi \int_{k_{\min }}^{k_{D}} k^{2} d k \delta(\omega-v \cdot k),
$$


which is reduced to: $D_{f}(\omega)=\frac{N_{x} N_{y} N_{z}^{f} a^{3}}{2 \pi^{2}} \frac{\omega^{2}}{v^{3}}$.

Debye frequency is determined from the normalization condition, which claims that the total number of phonon states must be equal to the number of atoms:

$$
\int_{\omega_{\min }}^{\omega_{f}^{t}} D_{f}(\omega) d \omega=N \Rightarrow \frac{N_{x} N_{y} N_{z}^{f} a^{3}}{2 \pi^{2} v^{3}} \int_{\omega_{\min }}^{\omega_{0}^{t}} \omega^{2} d \omega=N_{x} N_{y}\left(N_{z}^{f}+1\right),
$$

from which follows:

$$
\omega_{D}^{f}=\frac{\sqrt[3]{6 \pi^{2}}}{a} v \cdot \sqrt[3]{\frac{N_{z}^{f}+1}{N_{z}^{f}}+\frac{4}{3 \pi^{2}} \cdot \sin ^{3} \frac{\pi}{2\left(N_{z}^{f}+2\right)}}=\omega_{D}^{b} \cdot \sqrt[3]{\frac{N_{z}^{f}+1}{N_{z}^{f}+\frac{4}{3 \pi^{2}} \cdot \sin ^{3} \frac{\pi}{2\left(N_{z}^{f}+2\right)}}},
$$

where $\omega_{D}^{b}$ is Debye frequency for bulk structures, Eq. (10). By finding the ratio of the density of phonon states in ultrathin film and bulk structures at Debye frequencies, we are coming to the following conclusion:

$$
\frac{D_{f}\left(\omega_{D}^{f}\right)}{D_{b}\left(\omega_{D}^{b}\right)}=\frac{N_{z}^{f}}{N_{z}^{b}} \cdot\left(\frac{\omega_{D}^{f}}{\omega_{D}^{b}}\right)^{2}=\frac{N_{z}^{f}}{N_{z}^{b}} \cdot\left(\frac{N_{z}^{f}+1}{N_{z}^{f}}+\frac{4}{3 \pi^{2}} \cdot \sin ^{3} \frac{\pi}{2\left(N_{z}^{f}+2\right)}\right)^{\frac{2}{3}} \ll 1,
$$

because of $N_{z}^{f}<<N_{z}^{b}$. The consequences of this fact will be discussed in the Conclusion.

The internal energy of the ultrathin film is:

$$
U_{f}=\int_{\omega_{\min }}^{\omega_{D}^{t}} d \omega\left(\frac{\omega^{2} V}{2 \pi^{2} v^{3}}\right)\left(\frac{\hbar \omega}{e^{\frac{h \omega}{k_{B} T}}-1}\right)=9 N_{f} k_{B} T\left(\frac{T}{T_{D}^{f}}\right)^{3} \int_{x_{\min }}^{x_{D}^{t}} \frac{x^{3}}{e^{x}-1} d x
$$

where: $N_{f}=N_{x} N_{y}\left(N_{z}+1\right), \quad x_{\min }=\frac{\hbar \omega_{\min }}{k_{B} T}$ and $x_{D}^{f}=\frac{\hbar \omega_{D}^{f}}{k_{B} T}=\frac{T_{D}^{f}}{T}$. In the - physically most interesting - lowtemperature region $\left(x_{D}^{f} \rightarrow \infty\right)$, the expression for internal energy (27) becomes:

$$
U_{f}=9 N_{f} k_{B} T\left(\frac{T}{T_{D}^{f}}\right)^{3} \int_{x_{\min }}^{\infty} \frac{x^{3}}{e^{x}-1} d x=9 N_{f} k_{B} T\left(\frac{T}{T_{D}^{f}}\right)^{3}\left[\int_{0}^{\infty} \frac{x^{3}}{e^{x}-1} d x-\int_{0}^{x_{\min }} \frac{x^{3}}{e^{x}-1} d x\right]
$$

The first integral on the right has a solution:

$$
\int_{0}^{\infty} \frac{x^{3}}{e^{x}-1} d x=\frac{\pi^{4}}{15}
$$

$$
\left(e^{t}-1\right)^{-1}=\sum_{j=1}^{\infty} e^{-j t}
$$

so the expression (28) transforms into:

while the other is solved by series expansion of a sub-integral function:

$$
U_{f}=9 N_{f} k_{B} T\left(\frac{T}{T_{D}^{f}}\right)^{3}\left[\frac{\pi^{4}}{15}-\sum_{j=1}^{\infty} \int_{0}^{x_{\min }} x^{3} \cdot e^{-j \cdot x} d x\right]
$$

By multiple partial integration we obtain:

$$
U_{f}=9 N_{f} k_{B} \frac{T^{4}}{\left(T_{D}^{f}\right)^{3}}\left\{\frac{\pi^{4}}{15}+\sum_{j=1}^{\infty} \frac{1}{j} \cdot e^{-j \cdot x_{\min }}\left[\left(x_{\min }\right)^{3}+\frac{3}{j}\left(x_{\min }\right)^{2}+\frac{6}{j^{2}} x_{\min }+\frac{6}{j^{3}}\right]-\sum_{j=1}^{\infty} \frac{6}{j^{4}}\right\}
$$

Thermal capacitance calculated by one elementary cell of the crystal is:

$$
\begin{aligned}
C_{f}^{*} & =\frac{1}{N_{f}} \cdot \frac{\partial U_{f}}{\partial T}=\frac{12 \pi^{4}}{5} k_{B}\left(\frac{T}{T_{D}^{f}}\right)^{3}+9 k_{B}\left(\frac{T}{T_{D}^{f}}\right)^{3} \sum_{j=1}^{\infty} \frac{1}{j} \cdot e^{-j \cdot x_{\min }}\left[\frac{1}{j}\left(x_{\min }\right)^{4}+\right. \\
& \left.+\left(1+\frac{3}{j^{2}}\right)\left(x_{\min }\right)^{3}+\frac{6}{j}\left(1+\frac{1}{j^{2}}\right)\left(x_{\min }\right)^{2}+\frac{6}{j^{2}}\left(3+\frac{1}{j^{2}}\right) x_{\min }+\frac{24}{j^{3}}\right] .
\end{aligned}
$$


We introduce the following substitutions:

$$
\begin{array}{ll}
\frac{T}{T_{D}} \equiv \mathcal{T}, \frac{\omega_{\min }}{\omega_{D}} \equiv \Delta_{f}, & \frac{T}{T_{D}^{f}}=\frac{\omega_{D}}{\omega_{D}^{f}} \cdot \mathcal{T}=\frac{\mathcal{T}}{f\left(N_{z}\right)}, x_{\min }=\frac{T_{D}^{f}}{T} \cdot \frac{\omega_{\min }}{\omega_{D}^{f}}=\frac{\Delta_{f}}{\mathcal{T}} \\
\frac{\omega_{D}^{f}}{\omega_{D}}=\sqrt[3]{\frac{N_{z}^{f}+1}{N_{z}^{f}+\frac{4}{3 \pi^{2}} \cdot \sin ^{3} \frac{\pi}{2\left(N_{z}^{f}+2\right)}} \equiv f\left(N_{z}\right)} &
\end{array}
$$

on the basis of which we can write:

so the Eq. (30) becomes:

$$
\begin{aligned}
C_{f}^{*}= & \frac{12 \pi^{4}}{5} k_{B} \frac{\mathcal{T}^{3}}{f^{3}\left(N_{z}\right)}+9 k_{B} \frac{1}{f^{3}\left(N_{z}\right)} \sum_{j=1}^{\infty} \frac{1}{j} \cdot e^{-j \cdot \frac{\Delta_{A}}{\mathfrak{T}}}\left[\frac{1}{j} \frac{\Delta_{f}^{4}}{\mathfrak{T}}+\left(1+\frac{3}{j^{2}}\right) \Delta_{f}^{3}+\right. \\
& \left.+\frac{6}{j}\left(1+\frac{1}{j^{2}}\right) \Delta_{f}^{2} \mathfrak{T}+\frac{6}{j^{2}}\left(3+\frac{1}{j^{2}}\right) \Delta_{f} \mathcal{T}^{2}+\frac{24}{j^{3}} \mathfrak{T}^{3}\right] .
\end{aligned}
$$

Upon inclusion of the non-dimensional parameter: $C_{f}=\frac{C_{f}^{*}}{C_{0}}$, where $C_{0}=\frac{12 \pi^{4}}{5} k_{B}$, we get:

$$
\begin{aligned}
C_{f}= & \frac{\mathfrak{T}^{3}}{f^{3}\left(N_{z}\right)}+\frac{15}{4 \pi^{4}} \frac{1}{f^{3}\left(N_{z}\right)} \sum_{j=1}^{\infty} \frac{1}{j} \cdot e^{-j \cdot \frac{\Delta_{f}}{\mathfrak{T}}}\left[\frac{1}{j} \frac{\Delta_{f}^{4}}{\mathfrak{T}}+\left(1+\frac{3}{j^{2}}\right) \Delta_{f}^{3}+\right. \\
& \left.+\frac{6}{j}\left(1+\frac{1}{j^{2}}\right) \Delta_{f}^{2} \mathfrak{T}+\frac{6}{j^{2}}\left(3+\frac{1}{j^{2}}\right) \Delta_{f} \mathcal{T}^{2}+\frac{24}{j^{3}} \mathfrak{T}^{3}\right] .
\end{aligned}
$$

In order to get the graphical thermal capacitance display depending on the temperature in the low-temperature region, we will take the following parameter values:

- the speed of sound in crystal: $v \approx 10^{4} \mathrm{~m} / \mathrm{s}$;

- the lattice constant: $a \approx 10^{-10} \mathrm{~m}$;

- Debye frequency in bulk: $\omega_{D}^{b} \approx 4 \cdot 10^{14} \mathrm{rad} / \mathrm{s}$;

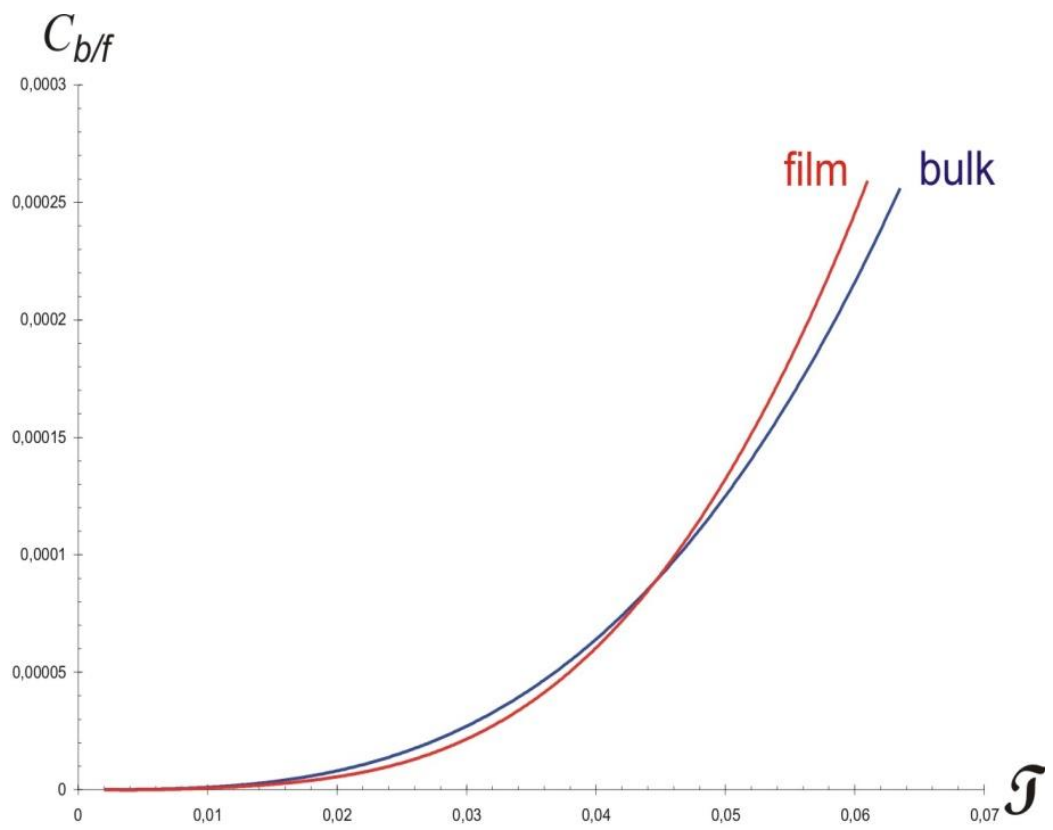

Figure 5. The comparative graph of the temperature dependence of thermal capacitance for the ultrathin film and bulk structure in the low temperature region

Slika 5. Uporedni grafički prikaz temperaturske zavisnosti toplotne kapacitivnosti za ultratanki film i balk u niskotemperaturskoj oblasti 


\section{DIFFUSION PROCESS OF PHONONS}

Bearing in mind that the nanostructures are mainly the highly anisotropic materials, the thermal processes in them are conveniently described using phonon diffusion tensor. Heat transfer in various nanostructured materials is introduced by means of tensor elements, giving all combinations of phonons diffusion rates [20]. It should be noted that the phonon diffusion tensor is also applicable to isotropic materials, but with the condition that only diagonal elements are different from zero [2123].

The diffusion tensor can be defined by the Kubo formula in the following manner [24-25]:

$$
D_{i j}(k)=\lim _{\delta \rightarrow 0+} \int_{0}^{\infty} d t e^{-\delta t}\left\langle\hat{v}_{i}(0) \hat{v}_{j}(t)\right\rangle,
$$

where $\hat{v}_{i}$ and $\hat{v}_{j}$ are velocity operators (in Heisenberg representation) of oscillations of molecules in crystal along the crystallographic direction $i, j \in(x, y, z)$, and $\delta$ is the perturbation parameter. $\left\langle\hat{v}_{i}(0) \hat{v}_{j}(t)\right\rangle$ are corresponding correlation functions. They will be calculated from their definition [15,26-27] (spectral theorem):

$$
\lim _{\delta \rightarrow+0}\left[G_{n \vec{n}}(\omega+i \delta)-G_{\vec{n} \dot{m}}(\omega-i \delta)\right]=\left(e^{\hbar \omega / \theta}-1\right) C_{\tilde{n} \dot{m}}^{G}(\omega),
$$

where $C_{\tilde{n} \dot{m}}^{G}(\omega)$ is the time - frequency Fourier's transform of the correlation function $C_{\hat{n} \dot{m}}^{G}(t)$. For $t=0$ the correlation functions represent the average values of the appropriate operators product.

In order to obtain the correlation functions that are part of the equation for diffusion coefficient $\left\langle\hat{v}_{i}(0) \hat{v}_{j}(t)\right\rangle$, we make use of the Green's function of momentum-momentum type $\left\langle\left\langle p_{i}(t) \mid p_{j}(0)\right\rangle\right\rangle$ since $\hat{v}_{i}=\frac{\hat{p}_{i}}{m}$ and $\hat{v}_{j}=\frac{\hat{p}_{j}}{m}$, and averaging will be done with big canonic ensemble, while the boundary conditions will be considered in the system of equations for crystalline films Green's function [25,28-30]. The appropriate correlation functions are:

$$
\left\langle p_{f}(t) p_{f}(0)\right\rangle=\frac{\hbar C}{\omega_{k}}\left(\frac{e^{-i \omega_{k} t}}{e^{h \omega_{k} / \theta}-1}-\frac{e^{i \omega_{k} t}}{e^{h \omega_{k} / \theta}-1}\right)
$$

Their inclusion in the formula for calculating the diffusion coefficient $D_{i j}(k)$ of the phonon subsystem gives:

$$
\begin{aligned}
& D_{i j}(k)=\lim _{\delta \rightarrow 0+} \frac{\hbar C}{M^{2} \omega_{k}} \times \\
& \times \int_{0}^{\infty}\left(e^{-\delta t} \frac{e^{-i \omega_{k} t}}{e^{\hbar \omega_{k}} / \theta-1}-e^{-\delta t} \frac{e^{i \omega_{k} t}}{e^{h \omega_{k} / \theta}-1}\right) d t,
\end{aligned}
$$

and finally:

$$
D_{i j}^{f}(k)=\left|i \frac{\hbar C}{M^{2}\left\langle\omega_{k}^{2}\right\rangle}\right| .
$$

From this result it can be seen that the diffusion tensor of phonon system is diagonal, that is $D_{i i}(k)=\frac{\hbar C}{M^{2}\left\langle\omega_{k}^{2}\right\rangle}$, and that the eigenvalues have higher values for the lower frequencies. The latter conclusion is very meaningful because it supports the macroscopic theories of heat conduction which states that diffusion coefficient is temperature independent. This fact and its consequences are very important in the theory of phonon engineering, especially regarding modern nanostructures in terms of achieving high-temperature superconductivity.

\section{CONCLUSION}

The main topic and the principal goal of this paper are to show the extent to which dimensional limitation of phonon movement affects the physical properties of nanostructures (ultrathin films, in particular, but the same theoretical approach can be applied to the other nanostructures). This idea forms the basis of the concept of phonon engineering, which is in the centre of scientific and engineering interest in recent years. The study is motivated by efforts to explain many unusual physical (mechanical, thermodynamic and optical) properties of contemporary quasi 2D materials, which have the great potential for applications in many fields of science and technology, as much as for improvement of human life in general.

Spatial limitation of acoustic and optical phonons in nanostructures significantly changes their properties in comparison with the massive materials. Their interactions are affected by the effects of dimensional confinement on the phonon modes in nanostructures, which results in a modification in the electron-phonon scattering rates, changes of the electrical, optical, acoustic and thermodynamic properties of the nanostructured materials, and phonon scattering on defects, boundaries and other phonons. Those modifications can be achieved by changing the lattice constant, i.e. dimensions of the ultrathin film, by inserting atoms of different kinds and by changing the external areas surrounding the 
boundary layers of the nanostructure (i.e. parameters $\varepsilon$ and $\gamma$ ).

It is a well-established fact that the phonons at Debye frequencies to the great extent affect the transport properties of materials. Since our results show that the density of phonon states in nanostructures is significantly lower than in the massive structures, the conclusion is that the nanofilm-structure is an inferior electrical and thermal conductor when compared to massive structures, providing there are no chemical and structural differences between them. On the other hand, it is also verified that the worse the conductor material is (under normal conditions), the better superconductor it becomes. Having this in mind, it can be concluded that in the spatially very limited structures the best superconducting properties can be expected, and also that the restriction of spatial dimensions of the structure plays a major role in improving superconducting properties of the material.

Regarding the thermodynamic properties, in the low-temperature region, the thermal capacitance of the film is lower than in massive structures, contrary to the middle-temperature region where it is the opposite. With an increase of film-thickness, the point of intersection of two curves moves towards lower temperatures. In addition, it is notable that the thermal capacitance of the film declines faster with a decrease of temperature than that of corresponding massive structure, and slowly increases with the rise of temperature to a certain upper temperature. Therefore, increasing the temperature of the film requires more thermal energy per mass unit in comparison to the bulk (with the identical crystallographic parameters).

\section{Acknowledgements}

This paper was in part supported by the Serbian Ministry of Education, Science and Technological Development (Grants: ON-171039 and TR-34019) and Ministry of Science and Technology of the Republic of Srpska (Grants: 19/6-020/961-15/16) as well as the Vojvodina Provincial Secretariat for Higher Education and Scientific-Research Development (Grants: 142451-2469/2017-01/02 and 142-451-2433/2016-01).

\section{REFERENCES:}

[1] A.A. Balandin, D.L.Nika (2012) Phononics in lowdimensional materials, Materials Today, 15 (6), 266275.

[2] V.M.Fomin, A.A.Balandin (2015) Phonon Spectrum Engineering in Rolled-up Micro- and NanoArchitectures, Applied Sciences, 5 (4), 728-746.

[3] A. Politano (2017) Spectroscopic Investigations of Phonons in Epitaxial Graphene, Critical Reviews in Solid State and Materials Science, 42 (2), 99-128.
[4] V.Sajfert, S.Jaćimovski, D.Popov, B.Tošić (2007) Statistical and dynamical equivalence of different elementary cells, Journal of Computational and Theoretical Nanoscience, 4 (3), 619-626.

[5] J.P.Šetrajčić, D.I.llić, S.K.Jaćimovski (2018) The influence of the surface parameter changes onto the phonon states in ultrathin crystalline films, Physica A: Statistical Mechanics and its Applications, 496, 434-445.

[6] C. Kittel (2004) Introduction to Solid State Physics, Wiley, New York.

[7] W. Jones, N.H. March (1973) Theoretical Solid State Physics, Vol. 2, Dover, New York.

[8] G. Rickayzen (1980) Green's Functions and Condensed Matter, Academic Press, London.

[9] M.Ohring (2001) Materials Science of Thin Films (2nd ed.), Academic Press, Boston.

[10] H.C.Chang, E.A.Demekhin (2002) Complex Wave Dynamics on Thin Films, Elsevier, Amsterdam.

[11] S.N. Karmakar, S.K.Maiti, J.Chowdhury (Eds.) (2007) Physics of Zero- and One-Dimensional Nanoscopic Systems, Springer-Verlag, Berlin Heidelberg New York.

[12] M.G.Cottam, D.R.Tilley (1989) Introduction to Surface and Superlattice Excitations, University Press, Cambridge.

[13] S.G.Davison, M.Steslicka (1996) Basic Theory of Surface States, Clarendon, Oxford.

[14] J.P.Šetrajčić, V.M.Zorić, S.M.Vučenović, D.Lj.Mirjanić, V.D.Sajfert, S.K. Jaćimovski, D.I.llić (2007) Phonon Thermodynamics in Crystalline Nanofilms, Materials Science Forum, 555, 291-296.

[15] J.P.Šetrajčić, D.Lj.Mirjanić, S.M.Vučenović, D.I.Ilić, B.Markoski, S.K.Jaćimovski, V.D. Sajfert, V.M. Zorić (2009), Phonon Contribution in Thermodynamics of Nano-Crystalline Films and Wires, Acta Physica Polonica A, 115 (4), 778-782.

[16] S.K.Jaćimovski, J.P.Šetrajčić, M.S.Jaćimovski, V.V.Stojanović (2014) Phonon contribution in thermodynamics and transport properties of ultrathin ceramic films, Acta Physica Polonica A, 126 (3), 811-819.

[17] J.P.Šetrajčić, S.K.Jaćimovski, V.D.Sajfert (2015) Phonon contribution to heat capacitance of nanolayered crystalline structures, Modern Physics Letters B, 29 (4), 1550008-15500086.

[18] J.P. Šetrajčić, V.D. Sajfert, S.K.Jaćimovski (2016) Fundamental preferences of the phonon engineering for nanostructural samples, Reviews in Theoretical Science, 4 (4), 353-401.

[19] J.P.Šetrajčić, S.K.Jaćimovski, V.D.Sajfert (2017) Diffusion of phonons through (along and across) the ultrathin crystalline films, Physica A, 486, 839-848.

[20] J.M. Ziman (2001) Electrons and Phonons: The Theory of Transport Phenomena in Solids, Oxford University Press, New York.

[21] J.M. Ziman (1999), Principles of the Theory of Solids, Cambridge University Press, Cambridge.

[22] R.J.B. Balaguru, B.G. Jeyaprakash, Lattice Vibrations, Phonons, Specific Heat Capacity, Thermal Conductivity, Joint Initiative of IITs and IISC - Funded by MHRD; http://www.nptel.ac.in /courses/115106076/Module\%209/Module\%209.pdf 
[23] L. Teylor, O. Heinonen (2002) A Quantum Approach to Condensed Matter Physics, Cambridge University Press, Cambridge.

[24] R. Kubo (1957) Statistical-Mechanical Theory of Irreversible Processes. I. General Theory and Simple Applications to Magnetic and Conduction Problems, Journal of the Physical Society of Japan, 12, 570-586.

[25] A.A. Abrikosov, L.P. Gorkov, I.E. Dzyaloshinski (1963) Methods of Quantum Field Theory in Statistical Physics, Dover Books, New York.

[26] G.Ghen, T.Zeng, T.Borca-Tasciuc, D.Song (2000) Phonon engineering in nanostructures for solidstate energy conversion, Materials Science and Engineering: A, 292 (2), 155-161.
[27] D.Zubarev, V.Morozov, G.Repke (1997) Statistical Mechanics of Nonequilibrium Processes, Vol.2, Akademie Verlag, Berlin.

[28] S.V.Tyablikov (1967) Methods in the Quantum Theory of Magnetism, Springer Science, New York.

[29] R.Kubo, M.Toda, N.Hashitsume (1998) Statistical Physics II - Quantum Field Theoretical Methods in Statistical Mechanics; Nonequilibrium Statistical Mechanics, Springer-Verlag, Berlin Heidelberg.

[30] V.D. Sajfert, J.P. Šetrajčić, S.K. Jaćimovski, B.S. Tošić (2005) Thermodynamic and kinetic properties of phonons in cylindrical quantum dots, Physica $\mathrm{E}$ : Low-dimensional Systems and Nanostructures, 25 (4), 479-491.

\section{IZVOD}

\section{UTICAJ FONONSKOG PODSISTEMA NA MEHANIČKE I TERMODINAMIČKE OSOBINE NISKODIMENZIONALNIH MATERIJALA}

Ovaj rad daje sažeti pregled uticaja fononog podsistema na mehanička i termodinamička svojstva niskodimenzionalnih materijala (konkretno, ultratankih filmova), koji su predmet istraživanja našeg tima već dugi niz godina. Ovakve studije postale su veoma važne za nauku o materijalima i inženjerstvo u poslednjih nekoliko godina, zbog velikog potencijala komercijalizacije novih dvodimenzionalnih struktura, kao što je grafen. Pokazano je kako kvantnomehanički efekat veličine utiče na promenu fizičkih osobina pomenutih struktura i detaljno su razmatrane posledice ovog uticaja. Teorijska analiza je sprovedena metodom dvovremenskih temperaturskih Grinovih funkcija, pomoću kojih su određeni spektri dozvoljenih energijskih stanja fonona, kao i termodinamičke karakteristike navedenih struktura. Procesi difuzije tretirani su pomoću Kubo formule, prilagođene fononskim podsistemima u nanostrukturima.

Ključne reči: Fononi, Grinove funkcije, ultratanki filmovi, specifična toplota, tenzor difuzije.

Naučni rad

Rad primljen: 31. 03. 2018.

Rad prihvaćen: 29. 04. 2018.

Rad je dostupan na sajtu: www.idk.org.rs/casopis

(c) 2018 Authors. Published by Engineering Society for Corrosion. This article is an open access article distributed under the terms and conditions of the Creative Commons Attribution 4.0 International license (https://creativecommons.org/licenses/by/4.0/) 